\title{
Efeitos do Uso Crônico do Amprenavir sobre a Prenhez da Rata Albina
}

\author{
Effects of Chronic Amprenavir Treatment on Rat Pregnancy
}

Dunya Rodrigues Mota ${ }^{1}$, Manuel de Jesus Simões ${ }^{1}$, Abês Mahmed Amed ${ }^{1}$

Adelino Moreira de Carvalho' ${ }^{1}$, Ricardo Martins de Oliveira-Filho², Luiz Kulay Júnior ${ }^{1}$

\section{RESUMO}

\begin{abstract}
Objetivo: avaliar o efeito do uso crônico do amprenavir sobre as implantações, reabsorções, fetos, placentas e mortalidade materna e fetal da rata albina.

Métodos: 5 grupos de ratas albinas EPM-1 Wistar, prenhes, foram usados: 2 controles, Contr 1 (controle do estresse) e Contr2 (controle do veículo), e três grupos experimentais, Exper1, Exper2 e Exper3, que receberam, respectivamente, 46, 138 e $414 \mathrm{mg} / \mathrm{kg}$ de peso de solução oral de amprenavir. Droga e veículo (propilenoglicol) foram ministrados por gavagem. Foram avaliados a evolução ponderal, número de implantações, de reabsorções, de fetos, de placentas e de óbitos intra-uterinos, o peso dos fetos e das placentas e malformações maiores. Foram retirados fragmentos de pulmões, rins, figado e intestinos para avaliação histopatológica.

Resultados: observou-se, no grupo Exper3, tendência a menor ganho de peso materno durante a prenhez ( $p=0,07)$, mas o amprenavir não causou efeitos deletérios sobre o conteúdo intra-uterino. O efeito citotóxico da droga revelou-se nas análises histopatológicas de vísceras das ratas prenhes e na taxa de mortalidade materna: 50\% nos grupos Exper1 e Exper2 e 70\% no grupo Exper3.

Conclusão: o amprenavir, em todas as doses administradas, mas especialmente na dose de $414 \mathrm{mg} / \mathrm{kg}$ de peso, mostrou ter efeito deletério sobre os pulmões, intestinos, rins e figado maternos e aumentou significantemente o percentual de mortalidade materna.
\end{abstract}

PALAVRAS-CHAVE: Drogas anti-retrovirais. AIDS. Estudo experimental. Malformações.

\section{Introdução}

Desde o seu reconhecimento como entidade nosológica, há cerca de duas décadas, a síndrome da imunodeficiência adquirida (SIDA, ou AIDS) representa um desafio médico, devido a sua alta taxa de mortalidade e ao número cada vez maior de individuos infectados.

Inicialmente conhecida como "doença dos homossexuais", desde 1993 a transmissão heterossexual passou a ser a principal forma de disseminação do vírus HIV no Brasil, aumentando consideravelmente o número de mulheres infectadas.

${ }^{1}$ Departamento de Obstetrícia - Universidade Federal de São Paulo - Escola Paulista de Medicina - São Paulo - SP - Brasil. ${ }^{2}$ Departamento de Farmacologia - Universidade de São Paulo - Instituto de Ciências Biomédicas, São Paulo - SP - Brasil. Correspondência:

Luiz Kulay Júnior

Rua Doutor Altino Arantes n ${ }^{\circ} 1000$, apto 203 - V. Clementino 04042-004 - São Paulo - SP

Fone: (11) 3285-4627

e-mail: kulay.toco@epm.br
Estima-se que, dos 42 milhões de infectados vivendo sob o binômio HIV/AIDS, 19,2 milhões correspondam a mulheres adultas ${ }^{1}$. No Brasil, no periodo de 1980 a 2002, o número de mulheres em idade fértil infectadas pelo HIV representou $85,5 \%$ dos casos de AIDS na população feminina. A incidência de gestantes soropositivas vem aumentando significativamente, segundo o Ministério da Saúde. No país existem cerca de $17 \mathrm{mil}$ gestantes HIV-positivas ${ }^{2}$.

O fenômeno de "feminização" da doença, principalmente em idade fértil, implica um número cada vez maior de crianças infectadas por meio da transmissão materno-fetal (TMF) ou transmissão vertical, sendo esta a principal via de infecção pelo HIV em crianças. No Brasil, até $2002,86,1 \%$ dos casos de infecção pelo HIV, verificados em menores de 13 anos, decorrem de TMF durante o ciclo gravídico-puerperal ${ }^{2}$.

Desde o estudo do Pediatric AIDS Clinical Group, conhecido como Protocolo 076 (ACTG 076), que demonstrou queda da TMF em $70 \%$ com a ad- 
ministração de zidovudina (AZT) à gestante ${ }^{3}$, os beneficios da terapia anti-retroviral vêm sendo amplamente estudados em associação com outras intervenções, como a cesárea eletiva e a orientação para supressão do aleitamento materno, a fim de reduzir a $\mathrm{TMF}^{4,5}$.

Embora inicialmente preconizada a monoterapia com AZT, há recomendações recentes de que as gestantes não devem ser privadas do uso de combinações de drogas potentes ${ }^{6}$, visto que atualmente esses esquemas estão relacionados a menor taxa de TMF ${ }^{7}$. Atualmente, cada vez mais, observa-se a tendência para manter o mesmo esquema que vinha sendo utilizado antes da gestação, exceto em casos de drogas reconhecidamente teratogênicas ${ }^{6,8}$.

O amprenavir é um anti-retroviral inibidor de protease de introdução recente na terapêutica, e não existem estudos bem controlados que avaliem o seu uso em gestantes ou suas repercussões sobre os conceptos. Deste modo, o presente trabalho averigua os possiveis efeitos adversos deste fármaco administrado durante toda a prenhez da rata albina.

\section{Material e Métodos}

Utilizamos ratas albinas (Rattus norvegicus albinus) da linhagem EPM-1 Wistar, virgens, com 90 dias de vida, pesando em torno de $230 \mathrm{~g}$, com regime de alimentação (ração Nuvital CR-1) e água ad libitum. Foram inicialmente acasaladas na proporção de três fêmeas para cada macho, por período de duas horas. O início da gestação (dia zero da prenhez) foi confirmado pelo encontro de espermatozóides na luz vaginal ${ }^{9}$. Cinqüenta ratas prenhes foram divididas, aleatoriamente, nos seguintes 5 grupos numericamente iguais: Contr1, animais não manipulados; Contr2, animais que receberam 0,5 mL de propilenoglicol; Exper1, Exper2 e Exper3, animais tratados, respectivamente, com 46,138 e $414 \mathrm{mg} / \mathrm{kg}$ de peso corporal de solução oral de amprenavir. O veículo e a droga foram administrados por gavagem, uma vez ao dia, sempre na parte da manhã, a partir do dia zero até o $20^{\circ}$ dia da prenhez.

O ganho de peso dos animais foi monitorado por meio de pesagem nos dias zero, $7^{\circ}, 14^{\circ}$ e $20^{\circ}$ de prenhez. Ao termo $\left(20^{\circ} \mathrm{dia}\right)$, todos os animais foram anestesiados com mistura de xilazina com ketamina (Rompun ${ }^{\circledR}$ e Francotar ${ }^{\circledR}$ ), 0,05 mL de cada uma, por via intraperitoneal. Após laparotomia e histerotomia, avaliaram-se os seguintes parâmetros: número de implantações, de reabsorções, de fetos vivos e mortos, bem como o peso dos fetos e placentas. Os fetos foram detalhadamente investigados sob lupa estereoscópica quanto à ocorrência de malformações maiores externas. Em seguida, as matrizes foram submetidas a necrópsia e retirados fragmentos de vários órgãos (pulmões, figado, intestinos e rins), os quais foram imediatamente mergulhados em formol a $10 \%$ e processados para análise histológica. Os cortes obtidos foram corados pela hematoxilina e eosina $(\mathrm{HE})$.

Os resultados, após análise de variância, foram analisados por comparações múltiplas pelo teste de Kruskal-Wallis. Para os cálculos, foi utilizado o software GraphPad Instat ${ }^{\circledR}$, versão 2.01.

\section{Resultados}

O ganho porcentual de peso das ratas foi avaliado no $7^{\circ}, 14^{\circ}$ e no $20^{\circ}$ dia de prenhez (Figura 1 ). Os resultados mostraram que o maior ganho de peso ocorreu no intervalo compreendido entre o $14^{\circ}$ e o $20^{\circ}$ dia. Embora não significante, o grupo que ganhou menos peso no último período foi o grupo Exper3.

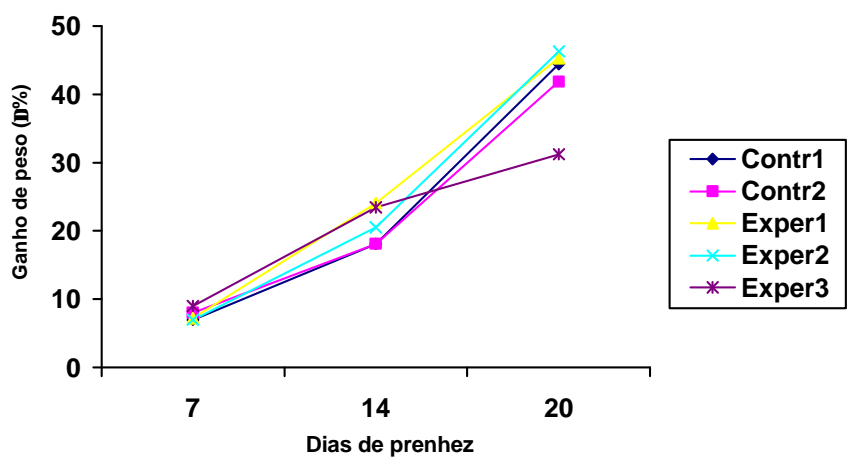

Figura 1 - Evolução do ganho de peso em ratas, controles (Contr1 = controle estresse e Contr2 = controle veículo) e tratadas com doses crescentes de amprenavir (Exper1 = $46 \mathrm{mg} / \mathrm{kg}$, Exper2 $=138 \mathrm{mg} / \mathrm{kg}$ e Exper3 $=414 \mathrm{mg} / \mathrm{kg})$ durante toda a prenhez $(\mathrm{n}=10 \mathrm{em}$ todos os grupos).

Quanto ao número de implantações verifica-se que no grupo Contr1 o número médio foi de 10,3 contra 10,3 no grupo Contr2 e variou de 10,0 a 12,2 nos grupos experimentais. A média de reabsorções foi de 0,3 (Contr1), 0,1 (contr2) e variou de 0 a 4 nos grupos experimentais. Os pesos dos fetos foram também semelhantes nos vários grupos: 4, 1 (Contr 1 e Contr2) e variou de 4,0 a 4,7 nos demais. O peso das placentas variou entre 0,6 e 0,7 em todos os grupos. Estes resultados mostram que não houve diferença significante entre os diversos grupos do experimento (Tabela 1). 
Tabela 1 - Eeitos do amprenavir sobre o número de implantações e de reabsorções, sobre o peso dos fetos e das placentas e sobre mortalidade materna\#.

\begin{tabular}{|c|c|c|c|c|c|}
\hline & Contr1 & Contr2 & Exper1 & Exper2 & Exper3 \\
\hline $\mathrm{N}^{0}$ de reabsorções & $0,3 \pm 0,21$ & $0,1 \pm 0,10$ & $0,2 \pm 0,20$ & - & $4,0 \pm 4,00$ \\
\hline Peso dos fetos $(\mathrm{g})$ & $4,1 \pm 0,10$ & $4,1 \pm 0,06$ & $4,0 \pm 0,11$ & $4,3 \pm 0,14$ & $4,7 \pm 0,75$ \\
\hline Peso das placentas (g) & $0,6 \pm 0,02$ & $0,6 \pm 0,02$ & $0,6 \pm 0,02$ & $0,7 \pm 0,03$ & $0,6 \pm 0,05$ \\
\hline Mortalidade materna (\%) & - & - & $50 \pm 16$ & $50 \pm 16$ & $70 \pm 15^{*}$ \\
\hline
\end{tabular}

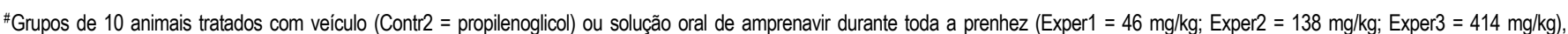
Contr1 = sem manipulação (controle de estresse).

${ }^{*} p<0,05$ em relação aos grupos Contr1 e Contr2.

Dados apresentados como média \pm erro padrão da média.

Os indices de mortalidade materna, nos grupos estudados, também estão expressos na Tabela 1. Houve expressiva mortalidade materna em todos os grupos tratados (50 $\pm 16 \%$ nos grupos Exper $1 \mathrm{e}$ Exper2, e $70 \pm 15 \%$ no grupo Exper3); contudo, apenas no grupo tratado com a dose maior (Exper3) o valor alcançou significância estatística.

A análise histológica mostrou comprometimento de alguns órgãos em vários graus de intensidade, à microscopia de luz, em todos os grupos experimentais, sendo mais intensa no grupo Exper3. Nos pulmões, observaram-se lesão alveolar difusa, congestão, edema e hemorragia; no intestino, verificaram-se necrose e descamação epitelial; no figado, observaram-se focos de necrose e esteatose e, nos rins, necrose tubular segmentar.

\section{Discussão}

O amprenavir é inibidor de protease viral com especificidade para o vírus HIV, cujo perfil de resistência a esse fármaco parece ser diferente do de outros congêneres, pois sua eficácia permanece por mais de 24 horas. Esta é a razão para dose única diária. Estudos clínicos mostram como principais efeitos colaterais do amprenavir: rash cutâneo, cefaléia, diarréia e perda de peso, mas pouco se sabe sobre as repercussões desse fármaco sobre a gestação. Neste trabalho, encontrou-se que a dose mais alta, ou seja, $414 \mathrm{mg} / \mathrm{kg}$ de peso corpóreo, apresentou efeitos indesejáveis como redução do ganho de peso e o aumento da mortalidade materna, numa primeira indicação para potenciais efeitos deletérios sobre a gestação da rata.

A dose de amprenavir administrada no grupo Exper1 foi proporcionalmente semelhante à recomendada para uso terapêutico em humanos, isto é, $46 \mathrm{mg} / \mathrm{kg}$ de peso corporal por dia. Os grupos Exper2 e Exper3 foram tratados com doses 3 e 9 vezes superiores à dose menor, em primeiro lugar porque o metabolismo hepático da rata estima-se ser três vezes mais eficiente do que aquele encontrado em humanos e a depuração renal (clearance) desses animais é duas vezes mais eficiente.

Os grupos que receberam amprenavir com doses de até $138 \mathrm{mg} / \mathrm{kg}$ (Exper1 e Exper2) apresentaram ganho de peso regular durante a gestação, não tendo havido ganho significantemente diferente do observado nos controles (Contr1 e Contr2). No grupo que recebeu $414 \mathrm{mg} / \mathrm{kg}$ (Exper3) os animais tiveram menor ganho de peso, em especial no último período da gestação. Observaram-se índices de $50 \%$ de mortalidade materna nos grupos Exper1 e Exper2, e de 70\% no grupo Exper3; além disto, houve grande número de reabsorções de conceptos nas ratas sobreviventes deste grupo. Tomados em conjunto, estes dados sugerem alto potencial tóxico do amprenavir para a prenhez da rata albina. Em humanos, o amprenavir está relacionado a intensas reações cutâneas, havendo também relatos de mortes em pacientes em uso desse fármaco, o que se estima em, aproximadamente, $1 \%$ dos $\operatorname{casos}^{10}$.

A taxa da passagem transplacentária do amprenavir, apesar de seu modesto peso molecular (505 Da), é extremamente baixa devido, provavelmente, ao bloqueio promovido pela glicoproteina $\mathrm{P}$, proteína de transporte transmembrana que existe em grande concentração junto à placenta; o mesmo tipo de bloqueio parece ocorrer também com o ritonavir, outro bloqueador de protease ${ }^{11-13}$. Tal fato pode explicar porque não temos observado efeitos deletérios do fármaco sobre o número de implantações e de reabsorções do experimento, como também sobre o peso dos fetos e das respectivas placentas nos grupos experimentais. A mesma ausência de efeitos no tocante ao conteúdo intra-uterino foi observada em estudo experimental similar usando ritonavir, provavelmente também em função da restrição da passagem transplacentária do fármaco promovida pela glicoproteina $\mathrm{P}^{14,15}$.

As lesões hepáticas e renais nos grupos tratados com amprenavir sugerem, em função da presumivel piora da metabolização e da depuração do fármaco, aumento de sua biodisponibilidade. 
A conseqüência imediata deste fato seria a potencialização de seus efeitos farmacológicos, com duas vertentes antagônicas: por um lado, em pacientes infectados haveria aumento da eficácia antiviral, e por outro, haveria deterioração das funções hepato-renais e aumento dos efeitos tóxicos sistêmicos próprios da droga (explicando, por exemplo, os efeitos observados neste trabalho sobre a mortalidade materna e sobre as reabsorções dos conceptos). Vale ressaltar, entretanto, que estas alterações podem ser espécie-específicas, e não devem ser transportadas, em primeira instância, para o ser humano. De qualquer forma, essas considerações podem contribuir para alertar o profissional médico para os cuidados especiais no monitoramento do uso dessa droga, especialmente no tocante a funções viscerais, particularmente quanto à função hepática e à função renal, promovendo a avaliação sistemática, periódica e freqüente do paciente, notadamente nas pacientes grávidas em eventual uso do amprenavir.

Embora já se soubesse que os inibidores de protease (indinavir, nelfinavir, ritonavir) podem desencadear resistência insulínica por inibição do transportador de glicose responsivo a insulina GLUT4 ${ }^{16,17}$, mais recentemente comprovou-se que o amprenavir apresenta potente efeito redutor da liberação de insulina sobre ilhotas pancreáticas isoladas ${ }^{18}$. Este fato agrava-se com a exposição prolongada e não é plenamente reversível após a retirada da droga ${ }^{17,18}$. Estes dados sugerem que exposição prolongada à terapia anti-retroviral de alta eficácia pode contribuir significativamente para o desenvolvimento de diabetes franco e têm duas conseqüências: primeiro, podem explicar, pelo menos em parte, os efeitos deletérios do amprenavir observados neste trabalho; segundo, chamam a atenção para o desenvolvimento de novas gerações de drogas anti-retrovirais que mantenham sua eficácia clínica em regimes de tratamento crônico sem causar efeitos metabólicos adversos, especialmente na gravidez.

\section{ABSTRACT}

Objective: to evaluate the chronic effects of amprenavir on implantations, reabsorptions, fetuses, placentae, and maternal and fetal mortality in the albino rat.

Methods: five groups of EPM-1 Wistar pregnant rats were used: two controls: Contr1 (control of stress) and Contr2 (drug vehicle control), and 3 experimental groups that were treated once a day throughout gestation with $46 \mathrm{mg} / \mathrm{kg}$ (Experl), $138 \mathrm{mg} / \mathrm{kg}$ (Exper2) and $414 \mathrm{mg} / \mathrm{kg}$ (Exper3) of oral solution of amprenavir. The drug and the vehicle (propyleneglycol) were administered by gavage. The evaluations included maternal body weight gain, number of implantations, reabsorptions, fetuses, placentae and of intrauterine deaths as well as fetal and placental mean weight and major malformations. Fragments of lungs, kidneys, liver and intestines were collected and prepared for histopathological evaluation.

Results: Exper3 group tended to show lesser maternal body weight gain during gestation $(P=0.07)$, but amprenavir did not affect the intrauterine contents. The cytotoxic effect of the drug was observed with regard to the histopathological analyses of pregnant rat viscerae and to the maternal mortality rate: $50 \%$ in Exper1 and Exper 2 groups, and 70\% in Exper3 group. Conclusion: amprenavir exerted adverse side effects on maternal lung, gut, kidney and liver, and significantly increased maternal mortality rates in all administered doses and especially at $414 \mathrm{mg} / \mathrm{kg}$.

KEY WORDS: Anti-retroviral drugs. Aids. Experimental study. Malformation.

\section{Referências}

1. World Health Organization. AIDS epidemic update. [online] 2002 Dec [cited 2003 Jan 21]: [45 screens]. Available from: URL: http://www.unaids.org/ worldaidsday /2002/press/update/epiupdate-2002en.doc

2. Ministério da Saúde. Coordenação Nacional DST/ AIDS. Dados epidemiológicos do Brasil. Bol Epidemiol Aids [online] 2002 [citado 20 jul 2003];16(2):[12 telas]. Disponivel em: URL: http:// www.aids.gov.br

3. Connor EM, Sperling RS, Gelber R, et al. Reduction of maternal-infant transmission of human immunodeficiency virus type 1 with zidovudine treatment. N Engl J Med 1994; 331:1173-80.

4. Bucceri AM, Somigliana E, Matrone R, et al. Combination antiretroviral therapy in $100 \mathrm{HIV}$-1-infected pregnant women. Hum Reprod 2002; 17:436-41.

5. The Italian Register for Human Immunodeficiency Virus Infection in Children. Determinants of mother-to-infant human immunodeficiency virus type 1 transmission before and after the introduction of zidovudine prophylaxis. Arch Pediatr Adolesc Med 2002; 156:915-21.

6. Centers for Disease Control and Prevention. US Public Health Service Task Force. Recommendations for use of antiretroviral drugs in pregnant HIV-1 infected women for maternal health and interventions to reduce perinatal HIV1 transmission in the United States [online]. Washington; 2002. [cited 2002 Out 30]: [159 screens]. Available from: URL: http: // www.aidsinfo.nih.gov/guidelines/perinatal/perinatal-083002.html 
7. Cooper ER, Charurat M, Mofenson L, et al. Combination antiretroviral strategies for the treatment of pregnant HIV-1 infected women and prevention of perinatal HIV-1 transmission. J Acquir Imunne Defic Syndr 2002; 29:484-94.

8. Taylor GP, Low-Beer N. Antiretroviral therapy in pregnancy: a focus on safety. Drug Saf 2001; 24:683702 .

9. Hamilton JB, Wolf JM. The effect of male hormone substance upon birth and prenatal development in the rat. Anat Rec 1938; 70:433-40.

10.Rotunda A, Hirsch RJ, Scheinfeld N, Weinberg JM. Severe cutaneous reactions associated with the use of human immunodeficiency virus medications. Acta Derm Venereol 2003; 83:1-9.

11.Smit JW, Huisman MT, van Tellingen O, Wiltshire HR, Schinkel AH. Absence or pharmacological blocking of placental P-glycoprotein profoundly increases fetal drug exposure. J Clin Invest 1999; 104:14417.

12.Huisman MT, Smit JW, Crommentuyn KM, et al. Multidrug resistance protein 2 (MRP2) transports HIV protease inhibitors, and transport can be enhanced by other drugs. AIDS 2002; 16:2295-301.
13. Marzolini C, Rudin C, Decosterd LA, et al. Transplacental passage of protease inhibitors at delivery. AIDS 2002; 16:889-93.

14. Carvalho AM. Ação crônica do ritonavir durante a prenhez da rata albina (Rattus norvegicus albinus, Rodentia, Mammalia). Repercussões sobre a performance reprodutiva e o resultado perinatal [tese]. São Paulo: Univ. Federal de São Paulo; 2003.

15.Mirochnick M, Dorenbaum A, Holland D, et al. Concentrations of protease inhibitors in cord blood after in utero exposure. Pediatr Infect Dis J 2002; $21: 835-8$.

16. Murata H, Hruz PW, Mueckler M. The mechanism of insulin resistance caused by HIV protease inhibitor therapy. J Biol Chem 2000; 275:20251-4.

17. Murata H, Hruz PW, Mueckler M. Indinavir inhibits the glucose transporter isoform Glut4 at physiologic concentrations. AIDS 2002; 16:859-63.

18.Koster JC, Remedi MS, Qiu H, Nichols CG, Hruz PW. HIV Protease inhibitors acutely impair glucosestimulated insulin release. Diabetes 2003; 52:1695700 .

Recebido em: 21/8/2003 Aceito com modificações em: 16/1/2004

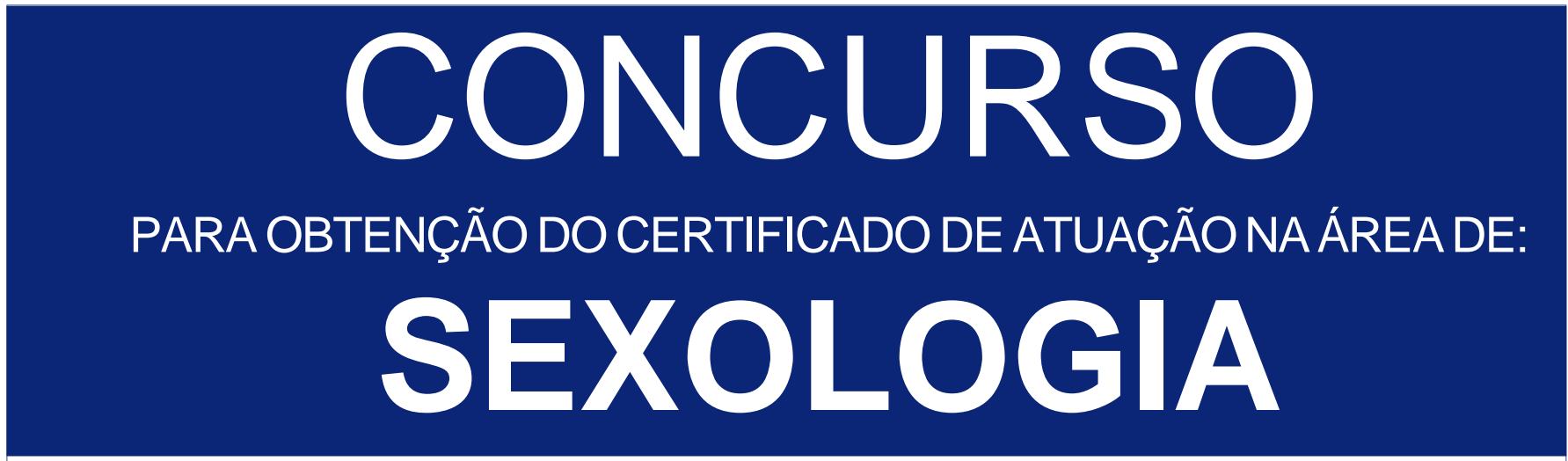

PERÍODO PARA ENVIO DO CURRÍCULUM:

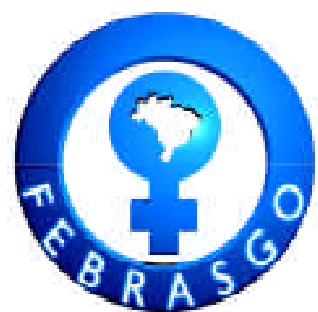

$15 / 04 / 2004$ A $14 / 06 / 2004$

\author{
EDITAL E FICHA DE INSCRIÇÃO: \\ Retirar na Home Page da FEBRASGO \\ www.febrasgo.org.br
}

ENVIAR PARA SECRETARIA EXECUTIVA DA FEBRASGO:

Av. das Américas, 8445 - sala 711

Barra da Tijuca - Rio de Janeiro - RJ

22793-081

Tel.: (0xx21) 2487-6336 